\title{
MedienPädagogik
}

Zeitschrift für Theorie und Praxis der Medienbildung www.medienpaed.com

\section{〈Ich wurde auf einem Bild markiert, da war so ein Mädchen und die war nackt)}

\section{Zur Notwendigkeit von visueller Kompetenz und Digitaler Souveränität}

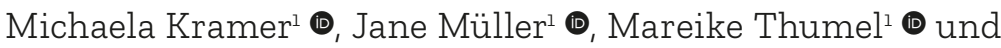 \\ Katrin Potzel ${ }^{1}$ (1) \\ ${ }^{1}$ Friedrich-Alexander-Universität Erlangen-Nürnberg
}

\section{Zusammenfassung}

Für Jugendliche sind (digitale) Bilder omnipräsent. Dabei werden sie auch mit sexualisiertem visuellem Material konfrontiert, welches in besonderer Weise einen kompetenten, sicheren und selbstbestimmten Umgang erfordert. Der Beitrag geht vor diesem Hintergrund der Frage nach, wie Jugendliche diese Handlungszusammenhänge wahrnehmen und welche Faktoren zu einer souveränen Praxis im Zuge ihres Sozialisationsprozesses beitragen. Nach einer thematischen Hinführung zum Feld der medienpädagogischen Jugendforschung werden hierzu die Ansätze der visuellen Kompetenz und der Digitalen Souveränität erläutert. Anschliessend wird anhand empirischen Datenmaterials aus einer explorativen Gruppendiskussions-Studie mit 7- und 8-Klässlerinnen und -klässlern illustriert, dass eine theoretische Sensibilisierung in diese beiden Richtungen fruchtbar ist, um das jugendliche Agieren in visualisierten, sexualisierten und datafizierten Medienumgebungen zu verstehen. Das Potenzial der konzeptionellen Verknüpfung visueller Kompetenz und Digitaler Souveränität wird abschliessend reflektiert und hierbei für ein Theorieverständnis plädiert, das sowohl bildtheoretische 
Prämissen als auch die Relationalität der Theoriebildung integriert. Ziel des Beitrags ist, auf empirischer und theoretischer Ebene ein Verständnis für das Aufwachsen in einer tiefgreifend mediatisierten Gesellschaft zu entwickeln, wobei vor dem Hintergrund der beschriebenen Relevanzen der Umgang mit Visualität und Sexualität in Social-Media-Kontexten im Zentrum steht.

\title{
'I was Tagged on a Picture: There was a Girl on it and she was Naked'. The Necessity of Visual Competence and Digital Sovereignty
}

\begin{abstract}
For young people, (digital) images are omnipresent. They are also confronted with sexualised visual material, which requires competent, safe and self-determined handling in a special way. Against this background, the article explores the question of how young people perceive these contexts of action and which factors contribute to a sovereign practice in the course of their socialisation process. After a thematic introduction to the field of media pedagogical youth research, the approaches of visual competence and digital sovereignty are explained. Subsequently, empirical data from an explorative group discussion study with $7^{\text {th }}$ and $8^{\text {th }}$ graders will be used to illustrate that a theoretical sensitisation in these two directions is fruitful in order to understand adolescent acting in visualised, sexualised and datafied media environments. Finally, the potential of conceptually linking visual competence and digital sovereignty is reflected, arguing for an understanding of theory that integrates both image-theoretical premises and the relationality of theory formation. The aim of the article is to develop an understanding of growing up in a mediatised society on an empirical and theoretical level. The focus lies on dealing with visuality and sexuality in social media contexts against the backdrop of the described relevancies.
\end{abstract}




\section{Zur Hinführung}

Digitale Bilder sind im Alltag Jugendlicher omnipräsent. Durch das Smartphone können Fotografien jederzeit und an jedem Ort produziert, bearbeitet und geteilt werden. Soziale Medien ermöglichen es zudem, Bilder anderer zu betrachten, zu liken, zu kommentieren und wiederum zu teilen. Diese Form der gegenwärtigen Bildpraxis ist eingebettet in einen gesellschaftlichen Transformationsprozess der Visualisierung (Reißmann 2015, 12), bei dem sich in mehreren historischen Schüben ein quantitativer und qualitativer Wandel des Umgangs mit Bildern vollzieht. Dabei nehmen Bilder spätestens seit der Digitalisierung einen zentralen Stellenwert im kommunikativen Handeln und in den Wirklichkeitserfahrungen von $\mathrm{He}$ ranwachsenden ein. Trotz ihrer scheinbar universellen Verständlichkeit sowie der leichten Handhabung von fotoproduzierenden und -distribuierenden Technologien sind gerade aufgrund der beschriebenen Omnipräsenz und Alltäglichkeit spezifische Kompetenzen notwendig, um sich in Bilderwelten zu orientieren. Die hier vorgestellte Studie offenbart darüber hinaus die grosse Bedeutung sexualisierter Bilder in den Lebenswelten Jugendlicher. Vor dem Hintergrund des Zusammenspiels von «Medialisierung und Sexualisierung» (Aigner et al. 2015) sind über eine problematisierende und moralisierende Annahme zur Veränderung sexueller Sozialisation durch (online-basierte) Medien hinaus die neuen Spielräume zu beachten, die das Internet Jugendlichen für ihre Sexualentwicklung eröffnet (Aigner et al. 2015, 8). Um in solchen herausfordernden Handlungszusammenhängen kompetent, selbstbestimmt und sicher zu agieren, sind - so die These des Beitrags - individuelle Fähigkeiten und überindividuelle Bedingungen von Bedeutung, die sich an der Schnittstelle von visueller Kompetenz und Digitaler Souveränität verorten lassen. Nach einer thematischen Hinführung zum Feld der medienpädagogischen Jugendforschung werden diese beiden Konzepte vorgestellt. Hieran schliesst die Darstellung der empirischen Studie an. Ausgewählte Themenbereiche verdeutlichen exemplarisch, wie beide theoretisch-konzeptionellen Perspektiven genutzt werden können, um das Medienhandeln Jugendlicher zu interpretieren und daran geknüpft Konsequenzen für pädagogische Handlungsfelder aber auch darüber hinaus - zu identifizieren. 


\section{Jugend in tiefgreifend mediatisierten Gesellschaften}

\subsection{Gesellschaftliche Transformation: Tiefgreifende Mediatisierung}

Da Kommunikation und Interaktion zunehmend medial bzw. digital vermittelt sind, ist der anhaltende Wandel der Gesellschaft durch Medien als sozialer Wandel erfahrbar (Thomas und Krotz 2008, 29). Unter Mediatisierung wird das Wechselverhältnis des Wandels von Medien und Kommunikation und des Wandels von Kultur und Gesellschaft verstanden, wobei beide Entwicklungen mit der Verbreitung technischer Kommunikationsmedien in Zusammenhang stehen (Krotz 2007, 12). Inzwischen sind nahezu alle Bereiche des menschlichen Lebens medial durchdrungen, über Medien verwirklicht und werden durch Medien gestaltet. Hepp spricht daran anschliessend von einer tiefgreifenden Mediatisierung (Hepp 2020). Zentrale Veränderungen sind die Ausdifferenzierung der Medientechnologie, deren wachsende Konnektivität, die Omnipräsenz digitaler (Mobil-)Kommunikation, eine beschleunigte Innovationsdichte und die Datafizierung des Medienhandelns (Hepp 2020, 28). Diese Trends lassen sich auch für Visualisierung als Subprozess tiefgreifender Mediatisierung spezifizieren (Kramer 2020, 66).

Das Verhältnis von Menschen zu ihrer Medienumgebung ist durch mediatisierte und visualisierte Interaktions- und Kommunikationsstrukturen komplex geworden. Durch Geräte und Strukturen setzen sich Menschen zueinander in Beziehung, präsentieren sich und teilen Informationen. Insbesondere Jugendliche sind «permanent online, permanent verbunden» (Vorderer et al. 2018) und nutzen digitale Medien ganz selbstverständlich (Jenkins, Ito, und Boyd 2016; Turkle 2011; Livingstone 2015). Das Medienhandeln findet hierbei meist in kommerziellen Strukturen (Livingstone 2008, 28; Brüggen et al. 2014) und stets vor dem Hintergrund rechtlicher Rahmenbedingungen statt. Jugendliche sind einerseits selbst Grundrechtstragende (z. B. Kunsturhebergesetz \$22) und müssen andererseits die Rechte Dritter beachten (z. B. Urheberrecht). 


\subsection{Sozialisation: Bildzentrierte Social-Media-Praktiken und die Bedeutung für die (sexuelle) Entwicklung Jugendlicher}

Sozialisationsprozesse können in tiefgreifend mediatisierten Gesellschaften nicht mehr ohne den konstituierenden und unhintergehbaren Einfluss digitaler Medien verstanden werden. Sozialisation wird dabei als wechselseitiger Prozess verstanden, bei dem Menschen vor dem Hintergrund ihrer biologischen Anlagen und ihrer sozialen und ökologischen Umwelt eine individuelle sowie soziale Identität entwickeln (Bauer und Hurrelmann 2015). Auch wenn die Ausbildung der Identität ein lebenslanger Prozess ist, findet er mit besonderer Intensität in der Adoleszenz statt (u. a. Kammerl 2014). Neben weiteren bedeutenden Entwicklungsbereichen sind der Aufbau von Peerbeziehungen und das Ausbilden einer sexuellen Identität (Göppel 2005) zentral. Die Bewältigung dieser Entwicklungsaufgaben hängt von kulturellen und historischen Begebenheiten, individuellen Voraussetzungen und persönlichen Unterstützungssystemen ab (Göppel 2005, 258). Hierbei suchen Jugendliche auch in Medienangeboten und in ihrem Medienhandeln nach Zukunftsentwürfen sowie Informationen über handlungsleitende Themen und soziale Fragen. Sie setzen Medien zielgerichtet ein: zur Alltagsbewältigung, zur Orientierung und in ihrem Streben nach Autonomie (Hugger und Tillmann 2020, 8ff.). Das Internet bietet in diesem Kontext Räume, in denen soziale, partnerschaftliche und sexuelle Erfahrungen gesammelt werden (Dekker und Thula 2017, 1034). Sie dienen zur Erprobung und Aushandlung von Geschlechter- und Körperbildern und werden als Ausdrucksmittel sexueller Intimität genutzt (Döring 2015, 31). Durch Mediatisierung und Kommerzialisierung wird das «Sexuelle zu einem von Bilder- und Zeichenwelten gefüllten Lebensbereich» (Ulbing 2020, 61).

Für kommunikatives Handeln sind insbesondere Messenger-Dienste relevant, über die unbegrenzt neben schriftlichen Nachrichten auch Sprachnachrichten, Videos und Bilder an Einzelpersonen oder Gruppen verschickt werden können. Instagram, Snapchat und TikTok sind weitere beliebte Apps, in denen Kommentare, Bilder und Videos geteilt, kommentiert und geliked werden. Durch die technisch einfachen sowie kostengünstigen Möglichkeiten der Bildaufnahme und -distribution hat eine Veralltäglichung der Fotopraxis stattgefunden (Kramer 2020). Dabei 
nutzen Jugendliche (visuelle) Medien in ihrem Alltag auf der Ebene des technischen Umgangs grösstenteils sehr kompetent. Sie eignen sich neue Medientechnologien besonders schnell und ohne empfundenen Aufwand an, und das erforderliche Wissen zur Nutzung der leicht handhabbaren Optionen der Bildproduktion, -bearbeitung und -distribution wird quasi nebenbei erworben (Kramer 2020). Das Rezipieren und das Verstehen von Fotografien scheinen aufgrund des universellen und sprachunabhängigen Zugangs zu ihnen auf den ersten Blick keiner dezidierten Kompetenzen zu bedürfen (Pietraß 2003). So können Bilder im Gegensatz zu Sprache ohne grössere Barrieren global verbreitet, wahrgenommen und decodiert werden. Dass je nach kulturellem Hintergrund denselben Bildern unterschiedliche Bedeutung zugewiesen werden kann, wird hierbei häufig übersehen.

Insgesamt können die Potenziale der Medienumgebung besser ausgeschöpft werden, wenn Strukturen und Machtverhältnisse kognitiv durchdrungen werden und Jugendliche die möglichen Mittel und Wege der Beteiligung erkennen. Hierbei kommt den Eltern eine zentrale Rolle zu, indem sie den Kindern ihr erstes Medienensemble vorgeben und über medienerzieherische Praktiken den Umgang mit diesem steuern (Kammerl et al. 2020; Hoffmann 2019; Shin und Kang 2016; Wagner et al. 2013). Wagner und Eggert stellen eine «Verfestigung von Ausgrenzungsmechanismen» $(2013,38)$ fest. So sind Wahrnehmung und Gestaltung von Interaktionsund Kommunikationsmöglichkeiten der Jugendlichen sowohl von deren Bildungshintergrund, dem sozioökonomischen Status und der Ressourcenausstattung der Familien abhängig als auch von individuellen Erfahrungen, dem Erleben von Kompetenz im Umgang mit digitalen Medien und den Unterstützungsstrukturen im sozialen Umfeld (u. a. Wagner und Eggert 2013, 38f.; sowie Eickelmann et al. 2019; Kutscher 2014; Boyd und Hargittai 2013; Hargittai 2010; Brüggen et al. 2019).

\section{Visuelle Kompetenz}

Auf die Frage, welche Fähigkeiten das Individuum braucht, um an der visuell mediatisierten Gesellschaft zu partizipieren und in dieser handlungsfähig zu sein, wurden im medienpädagogischen Diskurs, in der kulturellen Bildung und darüber hinaus bereits unterschiedliche konzeptionelle 
Antworten gegeben. Im deutschsprachigen Raum kursiert hierzu neben dem etabliertesten Konzept der visuellen Kompetenz (Hug und Kriwak 2010; Hermann, Stiegler, und Schlachetzki 2013) der vermehrt in kunstpädagogischen Kontexten zu findende Terminus der Bildkompetenz (Reichenbach und van der Meulen 2010). Dem übersichtlichen deutschen Diskurs steht eine Fülle konzeptioneller und empirischer Beiträge zu visual literacy im englischsprachigen Diskurs gegenüber, die sich auf verschiedene Literalitätskonzepte beziehen (Hug 2010, 60). Beiträge, die sich im Journal for Visual Literacy bündeln, adressieren hierunter vorrangig formale Bildungssettings und bilddidaktische Fragestellungen. Hiervon lässt sich das hiesige Theorieverständnis abgrenzen, in dem der Ausgangspunkt bei alltäglicher Bildkommunikation und medienkulturellen Praktiken im Kontext des jugendlichen Sozialisationsprozesses gewählt wird. So wird das Bild «im Zeitalter digitaler Medienkulturen und Netzwerkmedien (...) selbst zum Kommunikat in Alltag und Bildung sowie zum Angelpunkt von Werteaushandlungen und Zukunftsvorstellungen einer Gesellschaft (...). Ein dahingehend visualitätskompetenter Mensch weiss demnach um die kulturellen Hintergründe der Produktion, Distribution und Rezeption von digitalen sowie analogen Bildern und ist in der Lage, demgemäß zu handeln» (Missomelius 2017, 236).

Bildpraktiken werden in diesem Beitrag zunächst als präsentative Symbolpraktiken (Langer 1942/1984) verstanden, die sich von diskursiven Symboliken, wie etwa der gesprochenen Sprache, unterscheiden. Beide Formen der Vermittlung von Lebenserfahrungen und Gefühlen sind als «grundverschieden» (Langer 1942/1984, 99) anzusehen. Während die durch Diskursivität übertragenen Bedeutungen nacheinander dargestellt werden (ein Argument folgt auf das andere), bieten präsentative Symbole (Musik, Bilder, Körpersprache) den Rezipierenden ihre Bestandteile ganzheitlich und simultan dar. Über ein diskursiv-symbolisches und damit in gewisser Weise rationales Wissen über Medien hinaus sind somit ebenso die ganzheitlich-ästhetischen Bilder-Erfahrungen und daran gebundene Fähigkeiten im Umgang mit Bildern zentral (Mikos 2000). Insbesondere fotografische Bilder weisen dabei sowohl eine ikonische als auch eine symbolische Qualität auf (Mikos 2000, 8). Ihnen wird einerseits Authentizität 
zugeschrieben, da sie eine wahrnehmbare Ähnlichkeit zu einem Referenzobjekt aufweisen, andererseits sind sie zugleich Träger symbolischer Bedeutung. Im Anschluss an Mikos (2000) stellt sich die Unterscheidung zwischen ikonischer und symbolischer Qualität als wesentliches Merkmal visueller Kompetenz dar (Mikos 2000, 9). Dies geschieht nicht zwingend bewusst. So können Bilder auf symbolischer Ebene verstanden werden, ohne dass die ikonische Ebene noch bewusst reflektiert werden muss. In Social-Media-Kontexten werden beispielsweise massenhaft vermeintlich alltagsnahe Selfies von Stars oder Influencerinnen und Influencern hochgeladen, die Jugendliche dazu veranlassen, sie mimetisch und posenhaft nachzuahmen (Carnap, Flasche, und Kramer im Erscheinen). Den Heranwachsenden stellt sich dabei die Herausforderung, einerseits die spezifisch inszenierten Gestaltungsformen der Porträts zu erkennen und andererseits deren Einordnung als symbolischen Ausdruck eines Lifestyles, einer Kultur der Optimierung und der Vermarktung vorzunehmen. So geht es auch darum, eine kritische Haltung zu entwickeln, was ausschliesslich durch eine gewisse Distanzierung von der Norm erfolgen kann (Carnap, Flasche, und Kramer im Erscheinen).

Visuelle Kompetenz entwickelt sich ebenso wie der kompetente Umgang mit Sprache im Verlauf der Sozialisation und kann als «Ergebnis von medialen Lerngeschichten, die auf das Zusammenspiel von individuellen und gesellschaftlichen Lernprozessen gründen» (Mikos 2000, 11) verstanden werden. Die zentrale Bedeutung von Gesellschaftlichkeit kann an dieser Stelle mit Mannheims (1980) Konzept konjunktiver Erfahrungsräume präziser gefasst werden. Über die individuelle Peergroup hinaus werden demnach auch Erfahrungsräume relevant für visuelle Praktiken und zugrundeliegende Orientierungen, die sich nicht durch eine leibhaftige Anwesenheit der Mitglieder kennzeichnen. Auch solche Räume, in denen Menschen Ereignisse auf strukturidentische Weise erfahren, wie beispielsweise das «sozialisatorische Erleben von Genderverhältnissen》 (Bohnsack 2013) und ein ähnlich gelagertes medienbiografisches Wissen (Kramer 2020, 312ff.) bringen (kollektive) Praxis hervor. Im Hinblick auf die Frage, wie visuelle Kompetenz empirisch erfassbar werden kann, wird die Forderung des Einbezugs einer operativ-performativen Dimension virulent (Huber 2004; Burri 2008). So handelt es sich bei Kompetenz - was 
durchaus problematisch ist - stets um eine Attribution, einen «sozialen Beobachtungs-, Bewertungs- und Zuschreibungsprozess» (Huber 2004, 31). Über den Gegenbegriff der Performanz, der ein «öffentlich wahrnehmbares Verhalten» (Doelker 1997, 31) bezeichnet, wird eine mehr oder weniger kompetente Handlungspraxis bspw. an konkreten bildhaften Produkten ablesbar und zugänglich.

An dieser Stelle lässt sich festhalten, dass der Beitrag ein Verständnis visueller Kompetenz zugrunde legt, das die bildimmanente Fähigkeit der Differenzierung von ikonischen und symbolischen Bedeutungsebenen des Bildes ins Zentrum stellt. Die damit vorgenommene analytisch-isolierte Betrachtungsweise des Visuellen vermag die spezifisch ikonisch zu begründenden Produktions-, Wahrnehmungs- und Analysekompetenzen zum Vorschein zu bringen. So bieten Bilder und Bildpraktiken einen speziellen und von anderen Modalitäten zu unterscheidenden Zugang zur Welt und zum Selbst. Im alltäglichen Medienhandeln Jugendlicher befinden sich jedoch visuelle Elemente in dynamischer Verwobenheit verschiedener Modalitäten. Bild, Text und Audio sind im Rahmen von Alltagskommunikation kaum in Reinform vorzufinden (M. G. Müller und Geise 2015). Darüber hinaus sind Bilder in die medialen Verflechtungen der digitalen Netzwerke eingebunden, sodass über den symbolischen und ikonografischen Umgang mit Bildern hinaus auch technische und soziale Kompetenzen von Bedeutung sind wie das Wissen um die Algorithmizität von Plattformen sowie das geschickte Nutzen von Hashtags zur Wiederauffindbarkeit und Sichtbarkeit eines Beitrags (u. a. Jenkins und Purushotma 2009).

\section{Digitale Souveränität}

Immer wieder werden im Hinblick auf den Umgang mit digitalen Medien individuelle Fähigkeiten der Nutzenden ins Zentrum der Aufmerksamkeit gerückt. Der Ansatz visueller Kompetenz erweitert die Perspektive relevanter Fähigkeiten im Umgang mit digitalen Medien über die textsprachliche Ebene um die zentrale Ebene bildlicher Zugänge. Es zeigt sich jedoch darüber hinaus, dass der Umgang mit digitalen Medien nicht allein durch individuelle Fähigkeiten determiniert wird. Eine ganze Reihe exogener Faktoren ist hierbei von vergleichbarer Relevanz. Exogene und 
individuelle Faktoren beeinflussen sich dabei gegenseitig. Diese Relationalität adressiert der Ansatz der Digitalen Souveränität. Politische oder unternehmerische Fragestellungen beziehen sich schon seit längerem auf diesen. ${ }^{1}$ Zuletzt wird er zunehmend auch auf das (individuelle) Medienhandeln übertragen. Dabei sei der souveräne Umgang mit digitalen Medien entscheidend, um in einer tiefgreifend mediatisierten Welt individuelle Potentiale $\mathrm{zu}$ entfalten und an der Gesellschaft teilzuhaben (Jörissen 2019; Aktionsrat Bildung 2018).

Grundbegriffe der allgemeinen Pädagogik enthalten wesentliche Elemente klassischer Souveränitätsdefinitionen. So verweist etwa Klafki (2007, 19) auf verwandte Konzepte wie Selbstbestimmung, Emanzipation oder Autonomie, während Souveränität selbst lange Zeit keine bzw. nur eine untergeordnete Rolle spielte. Inzwischen wird der Begriff der Digitalen Souveränität im - medienpädagogisch relevanten - (weiteren) Kontext von Mediensozialisations-, Medienkompetenz- und Medienbildungsdiskursen, aber auch unabhängig davon aufgegriffen:

1. als Bildungsauftrag in Schulen (etwa Aktionsrat Bildung 2018; Meister 2013; Schulz-Zander 2001)

2. als Element von Medienkompetenz, etwa im Spannungsfeld zwischen sozialer Lebenswelt und Datenschutz, als Soziodigitale Souveränität bzw. soziale Haltung (Stubbe 2016, 2017) und als «digitales Bauchgefühl» (L.-S. Müller 2016) oder

3. als informationelle Selbstbestimmung (Friechsen und Bisa 2016; Groebel 2016).

Der Aktionsrat Bildung verweist in seiner Definition Digitaler Souveränität als Möglichkeit, «digitale Medien selbstbestimmt und unter eigener Kontrolle zu nutzen und sich an die ständig wechselnden Anforderungen

1 Auf Ebene der Politik geht es einerseits um die Digitale Souveränität Deutschlands bzw. der EU nach aussen, andererseits um das Setzen sinnvoller rechtlicher Rahmenbedingungen, um Digitale Souveränität von Unternehmen bzw. Bürgerinnen und Bürgern zu ermöglichen (u. a. Gueham 2017). Auf Ebene der Wirtschaft werden vor allem Fragen der Sicherheit und des Erfolgs von Unternehmen vor dem Hintergrund von ihnen eingesetzter Hard- und Software, aber auch dort arbeitender Mitarbeitender gestellt (u. a. Bundesministerium für Wirtschaft und Energie und Bundesministerium der Justiz und für Verbraucherschutz 2017). 
in einer digitalisierten Welt anzupassen»(Aktionsrat Bildung 2018, 12), lediglich auf individuelle Voraussetzungen und die «Bereitstellung entsprechender Technologien und Produkte» (Aktionsrat Bildung 2018, 12). Problematisch hieran ist nicht nur die (alleinige) Zuweisung eines Anpassungsbedarfes an gesellschaftliche, wirtschaftliche und technologische Entwicklungen an die Mediennutzenden, ohne bestehende Strukturen zu hinterfragen (insbesondere Niesyto 2021), sondern darüber hinaus die erneute (beinahe vollständige) Vernachlässigung personenexterner Rahmenbedingungen, welche das Medienhandeln lenken.

Eine systematische Ausarbeitung der Digitalen Souveränität und ihrer Relationen für die Ebene der Individuen steht noch aus. Sie wird hier exemplarisch für den Kontext jugendlichen Medienhandelns vorgenommen. Dabei definiert der Beitrag Digitale Souveränität als die Fähigkeiten und Möglichkeiten einer Person, kompetent, selbstbestimmt und sicher im Umgang mit oder in Abhängigkeit von digitalen Medien das eigene Leben zu gestalten. Er betont die Relationalität des Konzeptes und schlägt in einem ersten Schritt vor zunächst drei exogene Faktoren, die als besonders bedeutsam erachtet werden, in die Analyse einzubeziehen (J. Müller et al. 2020): soziale, technische und rechtliche Rahmenbedingungen. Gleichwohl ergab die bisherige Auseinandersetzung mit Digitaler Souveränität, dass diese nicht nur untereinander, sondern beispielsweise auch mit ökonomischen oder kulturellen Faktoren eng verbunden sind.

Auf der Ebene sozialer Bedingungen beeinflussen medientechnologische Entwicklungen nicht nur - wie bereits ausgeführt - Ressourcen und Fähigkeiten der Familien und darüber vermittelt die Kompetenz der Jugendlichen. Insbesondere Omnipräsenz und gesteigerte Konnektivität der Medien können auch dazu dienen, die Selbstbestimmung der Jugendlichen einzuschränken, wenn ihre Eltern etwa über technische Lösungen ihr Medienhandeln kontrollieren und damit elternfreie Entwicklungsräume der Jugendlichen schrumpfen (Marwick, Murgia-Diaz, und Palfrey 2010; Livingstone 2008; Boyd 2007). Verschärft wird diese Ausgangssituation durch die Peergroup der Jugendlichen, welche die zweite zentrale Bezugsgruppe für sie darstellt. Neben der Abgrenzung vom Elternhaus und der Erprobung einer eigenen Identität sind soziale Interaktionen mit den eigenen Peers ein wesentliches Entwicklungsbedürfnis Jugendlicher, welches 
diese auch in Onlinemedien bedienen wollen. Jugendliche orientieren sich «bei der Wahl von Messengern, Sozialen Online-Netzwerken und OnlineSpielen naheliegenderweise [sic!] an ihren Freundinnen und Freunden» (Brüggen et al. 2019, 33). So entstehen soziale Erwartungen. Der Wunsch nach sozialer Anerkennung kann zudem den Druck erhöhen, online immer mehr preiszugeben (Wagner, Brüggen, und Gebel 2010).

Die beschriebenen Entwicklungen illustrieren nicht nur die sozialen Bedingungen des Medienhandelns, sondern unterstreichen deren Verwobenheit mit technischen Voraussetzungen. Damit wird deutlich, dass technische Bedingungen des Medienhandelns als individuelle technische Ausstattung nicht ausreichend erfasst werden. Über die sozialen Implikationen hinaus spielt vor allem die anbieterseitige Gestaltung digitaler Medien eine zentrale Rolle. Auch diese wird von technologischen Trends moderiert. So werden User-Interfaces zum Teil zwar bedienfreundlicher, dahinterliegende Prozesse im Zuge einer immer stärkeren Ausdifferenzierung der Medien jedoch komplexer und unverständlicher (Matzner und Richter 2017; Dander und Aßmann 2015). Schwerer wiegt jedoch die zunehmende Datafizierung, in deren Folge ein Grossteil junger Menschen heute einen «digitalen Fußabdruck» hat, auch ohne aktiv etwas über sich selbst zu veröffentlichen (Livingstone 2017, 28). Entsprechende Profil- oder Nutzungsdaten entstehen nicht nur bei immer mehr alltäglichen Aktivitäten mit und in Onlinemedien, sie werden darüber hinaus immer häufiger aggregiert. Dies ermöglicht automatisierte und algorithmische Analysen, die in der Entstehung von Nutzungsprofilen, automatisierten Empfehlungen und personalisierten Newsfeeds bzw. personalisierter Werbung münden (Brüggen et al. 2019; Mascheroni, Ponte, und Jorge 2018; Mascheroni 2018; Wagner, Gebel, und Lampert 2013). Sie bergen damit ein hohes Potenzial für Manipulationen - insbesondere da die Plattformgestaltung weder Einsicht in noch Einfluss auf diese Prozesse zulässt (Wagner, Gebel, und Lampert 2013). Die so gegebenen technischen Bedingungen stehen dem selbstbestimmten Handeln Jugendlicher entgegen. Sie fühlen sich online zunehmend hilflos gegenüber der Datensammlung durch Unternehmen, aber auch durch Staaten (DeMooy 2017). 
Wenig Entgegenkommen lässt sich auch bei der Durchsetzung geltenden Rechts durch die Anbietenden digitaler Medientechnologien beobachten. Dabei macht die Rechtswissenschaft vor dem Hintergrund global agierender Technologieunternehmen eine Privatisierung der Rechtsdurchsetzung und eine Entmündigung der Bürgerinnen und Bürger aus (Hofmann 2019). Anbietende müssten «ihrer Verantwortung für eine sichere, unbeschwerte und altersgerechte Mediennutzung von Kindern und Jugendlichen gerecht werden» (Brüggen et al. 2019, 12; siehe auch Wisniewski 2018; Wagner, Brüggen, und Gebel 2010). Angelehnt an die Ergebnisse medienpädagogischer Analysen entwickeln Autorinnen und Autoren zudem verschiedene Vorschläge hierzu (etwa Livingstone et al. 2012; Wagner, Brüggen, und Gebel 2010). Demgegenüber schliessen «Anbieter die Nutzung populärer Online-Dienste durch Kinder und Jugendliche per AGB aus [...], obwohl diese Angebote zum etablierten Medienensemble der Kinderund Jugendmedienkultur zählen» (Brüggen et al. 2019, 15). Keine SocialMedia-Plattform bietet einen adäquaten Vorsorge-Schutz für Kinder und Jugendliche, um gefährdenden Inhalten zu entgehen (Jugendschutz.net 2020). Das hinter der technischen Verwirklichung liegende geltende Recht ist neben den dargestellten Regelungen rund um Urheber- und Persönlichkeitsrechte zentral. Auch dieses ist mit den Trends tiefgreifender Mediatisierung verschränkt. Ausdifferenzierung und Innovationsdichte verschärfen das Problem, dass die Rechtsprechung technologischen Entwicklungen hinterherhinkt (Gräf, Lahmann, und Otto 2018; Cap 2017; Brüggen et al. 2019). So ist etwa der Jugendmedienschutz an Angebotsstrukturen und Inhalten ausgerichtet statt am tatsächlichen Medienhandeln (Brüggen et al. 2019; Bulger et al. 2017). Die Rechtsprechung orientiert sich zudem an den Sorgen und Ängsten von Eltern und anderen Erwachsenen (Livingstone et al. 2014; Staksrud 2013; Drotner 1999; Bulger et al. 2017). Mit einer Perspektive auf Kinder und Jugendliche als passive, unschuldige, schutzbedürftige Wesen konzentrieren sich Regelungen vielfach auf Einschränkungen und Verbote.

Neben diesen am Schutz der Jugendlichen orientierten Regelungen gehören jedoch insbesondere Befähigung und Teilhabe zu den zentralen Rechten junger Menschen (United Nations 2021; Council of Europe 2018; United Nations Human Rights 1989). Eine Rechtssituation, die lediglich auf 
dem Ausschluss junger Menschen beruht, schützt nicht nur nicht vor Risiken, sondern nimmt auch Chancen (Livingstone 2017; Brüggen et al. 2019; Bulger et al. 2017). Will ein rechtlicher Rahmen Digitale Souveränität Jugendlicher ermöglichen und fördern, so muss er berücksichtigen, dass heute vielfältige Sozialisationsprozesse in den (privaten) Raum digitaler Angebote verlagert sind (Livingstone 2017) und sich dabei häufig dem Einblick Dritter entziehen (Brüggen et al. 2019). Jugendliche begegnen Risiken online in unterschiedlichen Rollen, nämlich als Rezipierende, Marktteilnehmende, Kommunizierende und/oder Produzierende (Hans-Bredow-Institut für Medienforschung 2014).

\section{Empirische Perspektive auf jugendliche Bildpraktiken}

\subsection{Projektkontext und methodisches Vorgehen}

Im Kontext eines interdisziplinären Forschungsverbundes, der sich an der Friedrich-Alexander-Universität Erlangen-Nürnberg (FAU) mit Digitaler Souveränität befasst, ${ }^{2}$ entstand eine explorative Studie zum Umgang Jugendlicher mit digitalen Daten. An fünf Schulen des Grossraums einer deutschen Grossstadt wurden im Sommer 2019 Gruppendiskussionen (Lamnek 2005) mit Jugendlichen erhoben. Ausgewählt wurde pro Schule je eine Klasse der achten Jahrgangsstufe (Alter der Jugendlichen war etwa 14 Jahre). Um ein möglichst kontrastreiches Sample zu generieren, wurden Mittelschulen und Gymnasien mit je einer Schule aus einem sozioökonomisch besser und einem schlechter gestellten Stadtteil rekrutiert. Je Gruppe diskutierten vier bis acht Jugendliche das Themenfeld Digitale Souveränität, operationalisiert als: Nutzung digitaler Medien, persönliche negative Erfahrungen, Erfahrungen Dritter, Wissen über nicht erlebte Gefahren, Handlungsstrategien zum Umgang mit diesen und ihr darauf bezogenes subjektives Empfinden. Die Gruppen wurden spontan - zumeist durch die Lehrperson - eingeteilt. Insgesamt nahmen 101 Jugendliche teil.

2 Weitere Informationen zu den Teilprojekten unter: www.digital-sovereignty. fau.de 
Der Leitfaden wurde nach einem Pre-Test teilstandardisiert. Die aufgezeichneten Gespräche wurden transkribiert, anonymisiert und mithilfe der qualitativen Inhaltsanalyse (Kuckartz 2018) ausgewertet. Während theoretische Vorannahmen zur Digitalen Souveränität bereits in die Entwicklung der Interviewleitfäden eingingen, kam der Fokus auf Aspekte visueller Kompetenz erst im Zuge der Datenanalyse hinzu. So zeigte sich während der Interpretationsarbeit eine auffällige Relevanzsetzung durch die Jugendlichen hinsichtlich des Umgangs mit digitalen Bildern - ohne dass diese im Vorhinein in der Erhebung angelegt war. Dies nahmen die Autorinnen zum Anlass die Daten unter der Fragestellung zu betrachten, welche Erfahrungen die Jugendlichen dezidiert mit Bildern in Social-Media-Kontexten machen. Hierzu wurden die beiden theoriebasierten Ansätze der Digitalen Souveränität und der visuellen Kompetenz in einen empirischen Auswertungszugang überführt. Besonderes Augenmerk wurde auf Textpassagen gelegt, in denen Zusammenhänge zwischen Kodierungen zu Aspekten Digitaler Souveränität und Aspekten visueller Kompetenz sichtbar wurden. ${ }^{3}$

\subsection{Ausgewählte Themenfelder der explorativen Studie}

Der Umgang mit Bildern in Social-Media-Kontexten stellte sich ausgehend von den Daten als besonders relevant heraus. Die Jugendlichen nehmen in ihrer alltäglichen Medienpraxis zahlreiche Herausforderungen wahr, denen sie mit spezifischen Strategien begegnen. Aus der Fülle der geschilderten Situationen wurden für den vorliegenden Beitrag drei Themenbereiche ausgewählt, die sich auf die Herausforderung einer Konfrontation mit sexualisiertem Bildmaterial zuspitzen lassen: von Dritten auf Bildern markiert werden, Penisbilder erhalten und Verbreitung privater Nacktbilder. Dass diese Themenfelder Phänomene aufgreifen, die von den Jugendlichen als problematisch wahrgenommen wurden, ist mit den Relevanzsetzungen der Interviewteilnehmenden zu begründen. Dies soll nicht darüber hinwegtäuschen, dass soziale Medien auch neue Freiräume und Potenziale für die sexuelle Sozialisation bieten können. Nachfolgend dient jeweils ein

3 Weitere Ergebnisse bei J. Müller et al. 2020. 
exemplarisch ausgewähltes Zitat aus den Gruppendiskussionen dazu, die Themenbereiche zu skizzieren und Anknüpfungspunkte visueller Kompetenz und Digitaler Souveränität aufzuzeigen.

\subsubsection{Themenbereich: Markiert werden}

Lukas: Zum Beispiel letztens, ich wurde auf einem Bild markiert, da war so ein Mädchen und die war nackt. Ich wurde darauf markiert einfach. Aber das war Fake-Account. [...] Man kann// nicht seinen Account/ na ja, man kann nix machen eigentlich, auch wenn dein Account privat ist. Du kannst trotzdem markiert werden. (GD Mittelschule 1)

Lukas beschreibt eine Situation, in der sein Account auf der Plattform Instagram mit einem Bild verlinkt wurde. Er rahmt dies verbal als eine nicht seltene negative Erfahrung, der gegenüber er für sich jedoch kaum Handlungsmöglichkeiten sieht. Das Bildmotiv beschreibt er konkret anhand der zu sehenden jungen Person weiblichen Geschlechts ohne Bekleidung, die er augenscheinlich nicht persönlich kennt. Eine explizite symbolische Einordnung als Pornografie nimmt er nicht vor, wodurch seine Äusserung als unaufgeklärt-naiv oder aber auch schüchtern-umschreibend interpretiert werden kann. Wie das Zitat verdeutlicht, werden in sozialen Medien Bilder ohne eigenes aktives Handeln von aussen an Jugendliche herangetragen. Die technischen Rahmenbedingungen der Plattformen erlauben es dabei, auf Bildern markiert zu werden. Die visuelle Selbstpräsentation der Jugendlichen auf Social Media ist damit nicht zwangsläufig ein individuell-autonomer Akt, der auf einer mehr oder weniger visuell kompetenten Handlungspraxis beruht. Sie hängt auch von schwer zu kontrollierenden Praktiken des Markierens und Verlinkens seitens anderer Akteurinnen und Akteure ab, wie sie als «machtvolle〉 Mechanismen in die Plattformen eingeschrieben sind. Zentral sind daneben auch die Rahmenbedingungen: Wer den Schüler markiert hat und wieso, bleibt im Gespräch unklar. Ob es sich um einen Scherz, Mobbing oder Werbung handelt - eine grosse Bandbreite an Möglichkeiten ist denkbar. Je nach Intention des oder der Markierenden wären bestimmte Rechtsbereiche betroffen. Gleichwohl 
ist die Situation nicht generell geregelt bzw. regelbar. Plattformbetreibende geben in ihren Nutzungsbedingungen zumeist nur die Möglichkeit, im Nachhinein Personen zu melden oder zu blockieren. Ein vorbeugender Schutz wird nur durch Nichtnutzung der Plattform erzielt.

\subsubsection{Themenbereich: Penisbilder erhalten}

Sexuelle Belästigung, auch durch den ungewollten Erhalt von Penisbildern, ist unter Jugendlichen, insbesondere bei Mädchen und jungen Frauen, ein weit verbreitetes Phänomen (u. a. Kohout 2018). Auch der Grossteil der teilnehmenden Mädchen berichtet davon, bereits ein- oder mehrmals entsprechende Bilder über Messenger gesendet bekommen zu haben. Exemplarisch soll hierfür der folgende Transkriptausschnitt stehen.

Emily: Also das ist auch bei Snapchat so, ne Freundin von mir, also allgemein so Mädchen, die bekommen die ganze Zeit so äh Bilder einfach von irgendwelchen Menschen.

Interviewerin:

Penissen.

$[\ldots]$

Aylin: Ja. Man bekommt das sehr oft. Also/

Emily: Also nur Mädchen so irgendwie.

Tanja: Das ist schon ekelhaft, so was.

(GD Gymnasium 1)

Die Interviewten schildern hier eine als «ekelhaft» bewertete Erfahrung, die sie einerseits als «Mädchen» verbinde und andererseits eine gewisse Normalität innerhalb ihres Gendermilieus aufweise. Hierbei wird die Messenger-App Snapchat genannt, für die eine kurze Sichtbarkeit der Bilder (bis zu zehn Sekunden bevor sie vom Gerät entfernt werden) im Sinne ephemerer Fotografie charakteristisch ist (Kofoed und Larsen 2016). Insbesondere solche 〈flüchtigen` Bilder bergen Gefahren, weil dieses vermeintliche Verschwinden das Verbreiten von riskanten Bildmotiven begünstigen kann (Lobinger und Schreiber 2017). In den Gruppendiskussionen werden sowohl Bekannte als auch Fremde und als «Peverslinge» oder «Pädophile» benannte Personen als Adressierende aufgeführt. Den Jugendlichen 
scheint weder klar zu sein, wer jeweils die Bilder absendet, noch warum sie diese erhalten. Klar ist ihnen hingegen, dass diese Bilder keinen ästhetischen Bezugsrahmen eröffnen, sondern als Symbol für einen sexuellen Übergriff stehen. In den Gruppendiskussionen wird deutlich, dass sie meist mit dem Blockieren des absendenden Accounts reagieren. Erneut ist ein präventiver Schutz vor entsprechenden Bildern seitens der Plattformen nicht möglich. Technisch können sich die Jugendlichen bei einigen Anbietenden schützen, indem sie ihr Profil auf «privat» schalten. Diese Möglichkeit ist jedoch nicht bei allen Plattformen gegeben. Auch schützt dies nicht zwangsläufig davor, entsprechende Bilder aus dem (weiteren) Bekanntenkreis zu erhalten. Den Betroffenen bleibt wiederum nur die Möglichkeit, die Accounts zu melden oder zu blockieren. Weitergehende Schritte werden seitens der Plattformbetreibenden nicht oder nur in Ausnahmefällen eingeleitet. Nach deutschem Recht stellt das ungefragte Versenden eines Penisbildes eine Straftat nach § 184 Nr. 6 StGB dar. Dennoch erstattete keine der Jugendlichen Strafanzeige. Dabei kann angenommen werden, dass ihnen diese Möglichkeit nicht bekannt war.

\subsubsection{Themenbereich: Verbreitung privater Nacktbilder}

Luca: Das ist nur dieses, äh, die Gefahr wenn so, keine Ahnung, Mädchen zum Beispiel jetzt irgendwelche Nacktfotos schicken, dass das halt wirklich rüber / alles rüber geschickt wird. Und halt wirklich oft geteilt wird.

(GD Gymnasium 1)

Ein weiteres relevantes Phänomen im Kontext jugendlicher Bild- und Social-Media-Praxis kommt im vorangegangenen Zitat zur Sprache: das unter dem Begriff Sexting diskutierte Senden privater Nacktfotografien. Jugendliche, die mit ihren Partnerinnen und Partnern Nacktbilder teilen, sehen sich zuweilen dem Vorwurf fehlender Medienkompetenz bzw. visueller Kompetenz gegenüber. Verschiedene Autorinnen und Autoren weisen vor dem Hintergrund Jugendlicher Identitätsbildung und fortschreitender Mediatisierung jedoch zu Recht darauf hin, dass das Erproben - auch der eigenen Sexualität und sexuellen Rolle - heute selbstverständlich in 
den digitalen Raum hineinreicht. So kann diese Praxis als funktionaler Bestandteil des Erwachsenwerdens und «normale» Identitätsarbeit im Sinne einer Selbst- und Körpererkundung verstanden werden (z. B. Döring 2014; Tillmann 2014). Dass hiermit gewisse «Fallstricke» (vgl. Tillmann 2014, 48) verbunden sind, sollte Anlass zu einer Erziehung Heranwachsender zur Verantwortung im sexuellen Handeln (Döring 2014, 62) und zur Förderung von Kunstfertigkeiten im Umgang mit Geschlechterungleichheiten und Doppelmoral genommen werden (Tillmann 2014).

Vielfältige Gründe veranlassen Jugendliche zum Weiterleiten von Bildern, die sie zuvor erhalten haben. Der Schaden lässt sich unabhängig vom Motiv kaum begrenzen. Versenden Adressatinnen und Adressaten von Nacktbildern diese ungefragt an Dritte, verstossen sie damit gegen geltendes Recht. Jedoch nützt dies den Geschädigten wenig, da sich einmal verbreitete Inhalte kaum mehr aus dem Internet entfernen lassen. In der Folge sind die Jugendlichen zum Teil extremem Mobbing und psychischem Druck ausgesetzt. Technisch haben Betroffene kaum Handhabe, die eigenen Bilder zurückzuverfolgen, geschweige denn zurückzuerhalten.

\section{Fazit: Diskursstränge zur konzeptionellen Verbindung}

Der vorliegende Beitrag reflektiert die Konzepte visueller Kompetenz und Digitaler Souveränität als Bezugspunkte in einer tiefgreifend mediatisierten und visualisierten Welt. Er systematisiert die Ansätze zunächst und illustriert sodann ihre empirische Anwendbarkeit am Beispiel von Gruppendiskussionen zur Auseinandersetzung mit sexualisierten Bildinhalten in sozialen Medien. Auf dieser empirischen Ebene kann an konkreten Phänomenen und Erfahrungen der alltäglichen Medienpraxis Jugendlicher aufgezeigt werden, dass zu deren Verständnis eine theoretische Sensibilisierung in beide Richtungen fruchtbar ist: Die Erfahrungen der Jugendlichen heben zum einen hervor, wie zentral die Auseinandersetzung mit visuellen Darstellungen und Inhalten für das heutige Aufwachsen ist. Zum anderen verdeutlicht der Mangel an Strategien, sich vor unerwünschten Erfahrungen mit sexualisierten Bildern zu schützen, nicht zwingend fehlende individuelle Fähigkeiten und Fertigkeiten, sondern zeigt vielmehr, 
dass exogene Faktoren ausserhalb des Einflussbereiches der Jugendlichen deren Medienhandeln moderieren. Zur Einordnung der gegenseitigen Befruchtung beider theoretischer Zugänge in den übergeordneten Theoriediskurs der Medienpädagogik werden zwei zentrale Diskursstränge aufgegriffen und diskutiert:

\subsection{Präsentativität}

Präsentativ-symbolische Formen des Selbstausdrucks sind für Jugendliche essenziell (Niesyto 2007). In tiefgreifend mediatisierten und individualisierten Gesellschaften dient die visuelle Selbstthematisierung über soziale Medien in besonderer Weise als Anstoss für Identitätsbildungsprozesse (Kramer 2020). Dennoch basieren Kompetenzmodelle, wie sie etwa im schulpolitischen Diskurs zugrunde gelegt werden, darauf, dass Wissen über die technischen Bedingungen von Medien erlangt wird. Die darüberhinausgehenden identitätsrelevanten Fähigkeiten zum präsentativen Ausdruck durch Bilder oder auch Musik werden hingegen nur marginal berücksichtigt (Mikos 2000). Auch mit dem Fokus auf Digitale Souveränität darf nicht ausser Acht gelassen werden, dass zwar ein sicherer und aufgeklärter Medienumgang zur gesellschaftlichen Teilhabe unabdingbar ist, dass jedoch auch die Medienpraktiken Jugendlicher gerade ihr Potenzial durch (irrationale, nicht-diskursive) ästhetische Erfahrungen in der Peergroup entfalten. Die Theorieperspektive visueller Kompetenz verhilft in diesem Kontext dazu, Bild und Text als verschiedene Zugänge zu Welt und Selbst anzuerkennen, denn die bildspezifische Doppeltheit der ikonischen und symbolischen Qualität macht den Umgang mit Bildern speziell und unterscheidet ihn vom Umgang mit Text bzw. Sprache. Während zu den Funktionsweisen von Sprache umfangreiches Wissen vorliegt, ist das Bildwissen durch die lange Zeit vorherrschende «pädagogische Marginalisierung des Bildes» (Missomelius 2017, 228) noch unzureichend. Das Potenzial des Bildes im Rahmen der praktischen Bildpädagogik im schulischen und ausserschulischen Kontext (Schlör 2017), der Auseinandersetzung mit Visualität in Lehr-Lernprozessen (Missomelius 2017) sowie auf der Ebene von Forschungsmethoden (Niesyto 2017; Kramer 2020) ist lange nicht ausgeschöpft. 


\subsection{Relationalität}

Das vorgestellte Konzept Digitaler Souveränität wählt einen Zugang, welcher neben relevanten individuellen Faktoren verstärkt exogene Einflüsse in den Mittelpunkt rückt. Der Ansatz unterstreicht damit nicht nur, dass Medienhandeln und sein Erfolg (aus jugendlicher, gesellschaftlicher, PeerPerspektive etc.) auch ausserhalb des Einflussbereichs der Handelnden liegt, sondern verweist zudem auf die Verwobenheit exogener und endogener Faktoren in diesem Prozess und berücksichtigt damit die Rolle tiefgreifender Mediatisierungsprozesse im Alltag allgemein und für das Aufwachsen Jugendlicher (J. Müller et al. 2020). Diese Relationalität und der systematische Einbezug von sozialen, technischen und rechtlichen Bedingungen sind konstitutiv für das hiesige Theorieverständnis Digitaler Souveränität. Insbesondere in Bezug auf jugendliche Social-Media-Praktiken, bei denen Bilder mitunter als riskante und sensible Daten zu verstehen sind, erscheint jedoch eine solche theoretische Perspektive erforderlich für das Verständnis der Handlungsmöglichkeiten und -begrenzungen im $\mathrm{Me}$ dienhandeln.

Abschliessend lässt sich festhalten, dass die Verknüpfung der theoretischen Konzepte visueller Kompetenz und Digitaler Souveränität in der vorgestellten empirischen Studie zum tieferen Verstehen der jugendlichen Medienpraktiken beiträgt. Hierdurch kann aufgezeigt werden, wo Handlungsbedarfe auf pädagogischer und rechtlicher sowie politischer Ebene bestehen. Die herausgegriffenen Themenfelder, die sich auf den Umgang mit sexualisierten Bildern in Social-Media-Kontexten beziehen, stellen Handlungszusammenhänge im Alltag Heranwachsender dar, in denen diese verschränkte Perspektive besonders fruchtbar erscheint. Sowohl der visualitätstheoretische als auch der relationale Zugang zu jugendlichem $\mathrm{Me}$ dienhandeln eröffnet Potenziale für Forschung und Praxis in diesem Feld. 


\section{Literatur}

Aigner, Josef Christian, Theo Hug, Martina Schuegraf, und Angela Tillmann. 2015. Medialisierung und Sexualisierung. Wiesbaden: Springer Fachmedien. https://doi.org/10.1007/978-3-658-06427-3.

Aktionsrat Bildung. 2018. Digitale Souveränität und Bildung: Gutachten. Unter Mitarbeit von Hans-Peter Blossfeld, Wilfried Bos, Hans-Dieter Daniel, Bettina Hannover, Olaf Köller, Dieter Lenzen, Nele McElvany et al. 1. Aufl. Münster: Waxmann. https://doi.org/10.25656/01:16569.

Bauer, Ullrich, und Klaus Hurrelmann. 2015. «Das Modell der produktiven Realitätsverarbeitung in der aktuellen Diskussion». Zeitschrift für Soziologie der Erziehung und Sozialisation 35 (2): 155-70. https://doi.org/10.3262/ZSE1502155.

Bohnsack, Ralf. 2013. «Dokumentarische Methode und die Logik der Praxis». In Pierre Bourdieus Konzeption des Habitus: Grundlagen, Zugänge, Forschungsperspektiven, herausgegeben von Alexander Lenger, Christian Schneickert, und Florian Schumacher, 175-200. Wiesbaden: Springer Fachmedien. https://doi. org/10.1007/978-3-531-18669-6_10.

Boyd, Danah. 2007. Why Youth Heart Social Network Sites: The Role of Networked Publics in Teenage Social Life. https://www.danah.org/papers/WhyYouthHeart. pdf. https://doi.org/10.31219/osf.io/22hq2.

Boyd, Danah, und Eszter Hargittai. 2013. "Connected and concerned: Variation in parents' online safety concerns». Policy \& Internet 5 (3): 245-69. https://doi. org/10.1002/1944-2866.POI332.

Brüggen, Niels, Stephan Dreyer, Christa Gebel, Achim Lauber, Raphaela Müller, und Sina Stecher. 2019. "Gefährdungsatlas. Digitales Aufwachsen. Vom Kind aus denken. Zukunftssicher handeln.». Unveröffentlichtes Manuskript. https://www.bzkj.de/bzkj/service/publikationen/gefaehrdungsatlas-digitalesaufwachsen-vom-kind-aus-denken-zukunftssicher-handeln--175506.

Brüggen, Niels, Eva Dirr, Mareike Schemmerling, und Ulrike Wagner. 2014. Jugendliche und Online-Werbung im Social Web. München. https://www.jff.de/ veroeffentlichungen/detail/jugendliche-und-online-werbung-im-social-web/.

Bulger, Monica, Patrick Burton, Brian O'Neill, und Elisabeth Staksrud. 2017. «Where policy and practice collide: Comparing United States, South African and European Union approaches to protecting children online». New Media \& Society 19 (5): 750-64. https://doi.org/10.1177/1461444816686325.

Bundesministerium für Wirtschaft und Energie, und Bundesministerium der Justiz und für Verbraucherschutz. 2015. "Maßnahmenprogramm "Mehr Sicherheit, Souveränität und Selbstbestimmung in der digitalen Wirtschaft": Herausforderungen und Handlungselemente für Gesellschaft, Wirtschaft und Verbraucher». https://www.bmwi.de/Redaktion/DE/Publikationen/Digitale-Welt/massnahmenprogramm-sicherheit-souveraenitaet-selbstbestimmung-digitale-wirtschaft.html. 
Burri, Regula Valérie. 2008. "Bilder als soziale Praxis: Grundlegungen einer Soziologie des Visuellen / Images as Social Practice: Outline of a Sociology of the Visual». Zeitschrift für Soziologie 37 (4): 342-58. https://doi.org/10.1515/zfsoz-2008-0404.

Cap, Clemens H. 2017. «Verpflichtung der Hersteller zur Mitwirkung bei informationeller Selbstbestimmung». In Informationelle Selbstbestimmung im digitalen Wandel, herausgegeben von Michael Friedewald, Jörn Lamla, und Alexander Roßnagel, 249-64. DuD-Fachbeiträge. Wiesbaden: Springer Vieweg. https:// doi.org/10.1007/978-3-658-17662-4.

Carnap, Anna, Viktoira Flasche, und Michaela Kramer. im Erscheinen. «Posieren oder Sich-Positionieren. Die Rekonstruktion von Haltungen in jugendlichen Social-Media-Praktiken». In Haltungen in der qualitativen Bildungs- und Biografieforschung, herausgegeben von Juliane Engel, Christine Demmer, Thorsten Fuchs, und Christine Wiezorek, o.S. Opladen: Barbara Budrich.

Council of Europe. 2018. «Recommendation CM/Rec(2018)7 of the Committee of Ministers to member States on Guidelines to respect, protect and fulfil the rights of the child in the digital environment». https://search.coe.int/cm/Pages/result_details.aspx?ObjectID=09000016808b79f7.

Dander, Valentin, und Sandra Aßmann. 2015. «Medienpädagogik und (Big) Data: Konsequenzen für die erziehungswissenschaftliche Medienforschung und -praxis». In Big Data und Medienbildung: Zwischen Kontrollverlust, Selbstverteidigung und Souveränität in der digitalen Welt, herausgegeben von Harald Gapski, 33-50. Schriftenreihe zur digitalen Gesellschaft NRW 3. Düsseldorf, München: kopaed. http://doi.org/10.25656/01:11634.

Dekker, Arne, und Koops Thula. 2017. «Sexting als Risiko? Zum konsensuellen und nichtkonsensuellen Versand persönlicher erotischer Fotos mittels digitaler Medien». Bundesgesundheitsblatt, Gesundheitsforschung, Gesundheitsschutz 60 (9): 1034-39. https://doi.org/10.1007/s00103-017-2595-9.

DeMooy, Michelle. 2017. «Rethinking Privacy Self-Management and Data Sovereignty in the Age of Big Data: Considerations for Future Policy Regimes in the United States and the European Union». 1. Auflage. http://doi. org/10.11586/2017009.

Döring, Nicola. 2014. "Consensual sexting among adolescents: Risk prevention through abstinence education or safer sexting?». Cyberpsychology 8 (1). https:// doi.org/10.5817/CP2014-1-9.

Döring, Nicola. 2015. «Smartphones, Sex und Social Media: Erwachsenwerden im Digitalzeitalter. Wie Jugendliche in Deutschland mit Smartphone, Apps und Social-Media-Plattformen umgehen». TELEVIZION 28 (1): 12-19. http://www. br-online.de/jugend/izi/deutsch/publikation/televizion/28_2015-1/DoeringSmartphones_Sex_und_Social_Media.pdf.

Doelker, Christian. 1997. Ein Bild ist mehr als ein Bild. Visuelle Kompetenz in der Multimedia-Gesellschaft. Stuttgart: Klett Cotta.

Drotner, K. 1999. «Dangerous media? Panic discourses and dilemmas of modernity». Paedagogica Historica 35: 593-619. https://doi.org/10.1080/0030923990350303. 
Eickelmann, Birgit, Wilfried Bos, Julia Gerick, Frank Goldhammer, Heike Schaumburg, Knut Schwippert, Martin Senkbeil, und Jan Vahrenhold, Hrsg. 2019. ICILS 2018 \#Deutschland: Computer- und informationsbezogene Kompetenzen von Schülerinnen und Schülern im zweiten internationalen Vergleich und Kompetenzen im Bereich Computational Thinking. Münster: Waxmann. https:// doi.org/10.25656/01:18166.

Friedrichsen, Mike, und Peter -J. Bisa, Hrsg. 2016. Digitale Souveränität. Vertrauen in der Netzwerkgesellschaft. Wiesbaden: Springer Fachmedien. https://doi. org/10.1007/978-3-658-07349-7_24.

Göppel, Rolf. 2005. Das Jugendalter: Entwicklungsaufgaben, Entwicklungskrisen, Bewältigungsformen. Pädagogik der Lebensalter 4. Stuttgart: Kohlhammer.

Gräf, Eike, Henning Lahmann, und Philipp Otto. 2018. «Die Stärkung der digitalen Souveränität: Wege der Annäherung an ein Ideal im Wandel». Unveröffentlichtes Manuskript, zuletzt geprüft am 12. Januar 2020. https://www. divsi.de/projekte/gesellschaft/digitaler-kodex/digitale-souveraenitaet/index. html.

Gueham, Farid. 2017. «Digital Souvereignty - Steps towards a new system of internet gouvernance». https://www.fondapol.org/en/study/digital-sovereignty-steps-towards-a-new-system-of-internet-governance/.

Hans-Bredow-Institut für Medienforschung an der Universität Hamburg. 2014. Aufwachsen mit digitalen Medien. Monitoring aktueller Entwicklungen in den Bereichen Medienerziehung und Jugendschutz. Hamburg. https://leibniz-hbi.de/ de/projekte/forschungs-monitoring-digitales-aufwachsen.

Hargittai, Eszter. 2010. «Digital $\mathrm{Na}(\mathrm{t})$ ives? Variation in Internet Skills and Uses among Members of the "Net Generation"». Sociological Inquiry 80 (1): 92-113. https://doi.org/10.1111/j.1475-682X.2009.00317.x.

Hepp, Andreas. 2020. Deep mediatization. Key Ideas in Media and Cultural Studies. London: Routledge. https://doi.org/10.4324/9781351064903.

Hermann, Thomas, Bernd Stiegler, und Sarah M. Schlachetzki. 2013. «Visuelle Kompetenz: Bilddidaktische Zugänge zum Umgang mit Fotografie». MedienPädagogik: Zeitschrift für Theorie Und Praxis Der Medienbildung 23 (Visuelle Kompetenz). https://doi.org/10.21240/mpaed/23/2013.00.00.X.

Hoffmann, Bernward. 2019. «Medien-Erziehungs-Kompetenz von Eltern im System Familie». In Digital Diversity: Bildung und Lernen im Kontext gesellschaftlicher Transformationen, herausgegeben von Holger Angenent, Birte Heidkamp, und David Kergel, 103-17. Diversität und Bildung im digitalen Zeitalter. https://doi.org/10.1007/978-3-658-26753-7_7.

Hofmann, Franz. 2019. Digitale Souveränität: Perspektive der Privatrechtswissenschaft. Erlangen. Working Paper Emerging Fields Initiative (Digital Sovereignty〉 (Friedrich-Alexander-Universität Erlangen-Nürnberg).

Huber, Hans Dieter. 2004. «Visuelle Performativität». In Visuelle Netze. Wissensräume in der Kunst, herausgegeben von Hans D. Huber, Bettina Lockemann und Michael Scheibel, 31-37. Ostfildern-Ruit: Hatje Cantz. 
Hug, Theo. 2010. «Visuelle Kompetenz, Medienkompetenz und «New Literacies» - Konzeptionelle Überlegungen in einer pluralen Diskurslandschaft». In Visuelle Kompetenz. Beiträge des interfakultären Forums Innsbruck Media Studies, herausgegeben von Theo Hug, und Andreas Kriwak, 54-74. Innsbruck: innsbruck university press. https://doi.org/10.25969/mediarep/14859.

Hug, Theo, und Andreas Kriwak, Hrsg. 2010. Visuelle Kompetenz. Beiträge des interfakultären Forums Innsbruck Media Studies. Innsbruck: innsbruck university press. https://doi.org/10.25969/mediarep/14859.

Hugger, Kai-Uwe, und Angela Tillmann. 2020. «Kindheit und Jugend im Wandel». In Handbuch Kindheits- und Jugendforschung, herausgegeben von Heinz-Hermann Krüger, Cathleen Grunert, und Katja Ludwig, 1-18. Wiesbaden: Springer Fachmedien. https://doi.org/10.1007/978-3-658-24801-7_30-1.

Jenkins, Henry, Mizuko Ito, und Danah Boyd. 2016. Participatory culture in a networked era: A conversation on youth, learning, commerce, and politics. Cambridge: Polity Press. https://doi.org/10.17192/ep2016.4.6302.

Jenkins, Henry, und Ravi Purushotma. 2009. Confronting the Challenges of Participatory Culture: Media Education for the 21st Century. The John D. and Catherine T. MacArthur Foundation Reports on Digital Media and Learning. Cambridge, Mass. The MIT Press. https://www.macfound.org/press/grantee-publications/ white-paper-confronting-the-challenges-of-participatory-culture-mediaeducation-for-the-21st-century-by-henry-jenkins.

Jörissen, Benjamin. 2019. «Die Digitalisierung und ihr Einfluss auf die Gesellschaft». In Digitale Kompetenzen in der Jugendarbeit, herausgegeben von Deutsche Telekom Stiftung, 7-17.

Jugendschutz.net. 2020. «Bericht 2019: Jugendschutz im Internet: Risiken und Handlungsbedarf». https://www.jugendschutz.net/jahresberichte/index. html.

Kammerl, Rudolf. 2014. «Enkulturationshilfen in der digitalen Gesellschaft Diskurse als/oder Orientierung?». In Jahrbuch Medienpädagogik 11, herausgegeben von Rudolf Kammerl, Alexander Unger, Petra Grell, und Theo Hug, 15-33. Wiesbaden: Springer Fachmedien. https://doi.org/10.1007/978-3-658-064624_2.

Kammerl, Rudolf, Jane Müller, Claudia Lampert, Marcel Rechlitz, und Katrin Potzel. 2020. «Kommunikative Figurationen - ein theoretisches Konzept zur Beschreibung von Sozialisationsprozessen und deren Wandel in mediatisierten Gesellschaften?». In Bewegungen, herausgegeben von Isabell van Ackeren, Helmut Bremer, Fabian Kessl, Hans C. Koller, Nicolle Pfaff, Caroline Rotter, Dominique Klein, und Ulrich Salaschek, 377-88. Opladen, Berlin, Toronto: Barbara Budrich. https://doi.org/10.2307/j.ctv1oh9fjc.30.

Klafki, Wolfgang. 2007. Neue Studien zur Bildungstheorie und Didaktik. Zeitgemäße Allgemeinbildung und kritisch-konstruktive Didaktik. Weinheim: Beltz.

Kofoed, Jette, und Malene Charlotte Larsen. 2016. "A snap of intimacy: Photosharing practices among young people on social media». First Monday 21 (11): 1-16. https://doi.org/10.5210/FM.V21I11.6905. 
Kohout, Raphaela. 2018. «Sexuelle Belästigung und Gewalt im Internet in den Lebenswelten der 11-bis 18-Jährigen: durchgeführt vom Institut für Jugendkulturforschung im Auftrag von SOS-Kinderdorf Österreich und Rat auf Draht». https://jugendkultur.at/publikationen/online/.

Kramer, Michaela. 2020. Visuelle Biografiearbeit: Smartphone-Fotografie in der Adoleszenz aus medienpädagogischer Perspektive. Lebensweltbezogene Medienforschung. Baden-Baden: Nomos. https://doi.org/10.5771/9783748910237.

Krotz, Friedrich. 2007. Mediatisierung: Fallstudien zum Wandel von Kommunikation. Wiesbaden: VS Verlag für Sozialwissenschaften. https://doi.org/10.1007/978-3531-90414-6.

Kuckartz, Udo. 2018. Qualitative Inhaltsanalyse. Methoden, Praxis, Computerunterstützung. 4. Auflage. Grundlagentexte Methoden. Weinheim, Basel: Beltz Juventa.

Kutscher, Nadia. 2014. «Soziale Ungleichheit». In Handbuch Kinder und Medien, herausgegeben von Angela Tillmann, Sandra Fleischer, und Kai-Uwe Hugger, 101-12. Wiesbaden: Springer VS. https://doi.org/10.1007/978-3-531-18997-0_7.

Lamnek, Siegfried. 2005. Gruppendiskussion: Theorie und Praxis. Weinheim: Beltz PVU.

Langer, Susanne K. 1942/1984. Philosophie auf neuem Wege. Frankfurt a. M.: Fischer.

Livingstone, Sonia. 2008. "Taking risky opportunities in youthful content creation: teenagers' use of social networking sites for intimacy, privacy and self-expression». New Media Society 10 (8): 393-411. https://doi. org/10.1177/1461444808089415.

Livingstone, Sonia. 2015. "Children's internet culture: Power, change and vulnerability in twenty-first century childhood». In The Routledge international handbook of children, adolescents and media, herausgegeben von Dafna Lemish, 111-19. Routledge international handbooks. London: Routledge. https://doi. org/10.4324/9780203366981-26.

Livingstone, Sonia. 2017. "Children's and Young People's Lives Online». In Online Risk to Children: Impact, Protection and Prevention, herausgegeben von Jon Brown, 23-36. Wiley Child Protection and Policy Ser. Newark: John Wiley \& Sons Incorporated. https://doi.org/10.1002/9781118977545.ch2.

Livingstone, Sonia, Lucyna Kirwil, Cristina Ponte, und Elisabeth Staksrud. 2014. «In their own words: What bothers children online?». European Journal of Communication 29 (3): 271-88. https://doi.org/10.1177/0267323114521045.

Livingstone, Sonia, Kjartan Ólafsson, Brian O'Neill, und Veronica Donoso. 2012. «Towards a better internet for children: findings and recommendations from EU Kids Online to inform the CEO coalition. EU Kids Online». Unveröffentlichtes Manuskript, zuletzt geprüft am 5. August 2020. http://eprints.lse. ac.uk/id/eprint/44213. 
Lobinger, Katharina, und Maria Schreiber. 2017. «Photo Sharing». In Handbuch Visuelle Kommunikationsforschung, herausgegeben von Katharina Lobinger, 1-22. Wiesbaden: Springer Fachmedien Wiesbaden. https://doi.org/10.1007/978-3658-06738-0_19-1.

Marwick, Alice E., Diego Murgia-Diaz, und John G. Palfrey. 2010. «Youth, Privacy and Reputation (Literature Review)». https://ssrn.com/abstract=1588163.

Mascheroni, Giovanna. 2018. "Datafied childhoods: Contextualising datafication in everyday life». Current Sociology 68 (6): 798-813. https://doi. org/10.1177/0011392118807534.

Mascheroni, Giovanna, Cristina Ponte, und Ana Jorge, Hrsg. 2018. Digital Parenting: The Challenges for Families in the Digital Age. Yearbook / The International Clearinghouse on Children, Youth and Media 2018. http://urn.kb.se/resolve?u rn=urn:nbn:se:norden:org:diva-5398.

Matzner, Tobias, und Philipp Richter. 2017. «Die Zukunft der informationellen Selbstbestimmung». In Informationelle Selbstbestimmung im digitalen Wandel, herausgegeben von Michael Friedewald, Jörn Lamla, und Alexander Roßnagel, 319-23. DuD-Fachbeiträge. Wiesbaden: Springer Vieweg. https://doi. org/10.1007/978-3-658-17662-4.

Meister, Dorothee M. 2013. «Vermittlung von Medienkompetenz in der Praxis für Kinder und Jugendliche: Schule». In Medienkompetenzförderung für Kinder und Jugendliche. Eine Bestandsaufnahme, herausgegeben von Bundesministerium für Familie, Senioren, Frauen und Jugend, 46-52. https://www.gmk-net.de/ wp-content/uploads/2018/07/medienkompetenzbericht_2013.pdf.

Mikos, Lothar. 2000. «Ästhetische Erfahrung und visuelle Kompetenz: Zur Erweiterung der diskursiven Medienkompetenz um präsentative Elemente». MedienPädagogik: Zeitschrift für Theorie Und Praxis Der Medienbildung I (Medienkompetenz): 1-16. https://doi.org/10.21240/mpaed/01/2000.03.19.X.

Missomelius, Petra. 2017. «Medienpädagogische Aufgabenfelder hinsichtlich der Visualität im digitalen Zeitalter. Desiderate im Umgang mit visuellen Medienkulturen». MedienPädagogik: Zeitschrift für Theorie Und Praxis Der Medienbildung 27 (Spannungsfelder \& blinde Flecken): 226-38. https://doi.org/10.21240/ mpaed/27/2017.04.27.X.

Müller, Jane, Ina Mareike Thumel, Katrin Potzel, und Rudolf Kammerl. 2020. «Digital Sovereignty of Adolescents». Medien Journal. Zeitschrift für Kommunikationskultur (Special Issue 02/2020 "Digital Culture, New Media and Youth"): 30-40. https://doi.org/10.24989/medienjournal.v44iı.1926.

Müller, Lena-Sophie. 2016. «Das digitale Bauchgefühl». In Digitale Souveränität: Vertrauen in der Netzwerkgesellschaft, herausgegeben von Mike Friedrichsen und Peter -J. Bisa, 267-85. Wiesbaden: Springer Fachmedien. https://doi. org/10.1007/978-3-658-07349-7_24.

Müller, Marion G., und Stephanie Geise. 2015. Grundlagen der Visuellen Kommunikation. 2. Aufl. 2414. Konstanz, München: UVK. 
Niesyto, Horst. 2007. «Eigenproduktionen mit Medien als Gegenstand medienpädagogischer Praxisforschung». In Jahrbuch Medien-Pädagogik 6, herausgegeben von Werner Sesink, Michael Kerres, und Heinz Moser, 222-45. Wiesbaden: VS Verlag für Sozialwissenschaften. https://doi.org/10.1007/978-3-531-90544O_11.

Niesyto, Horst. 2017. «Visuelle Methoden in der medienpädagogischen Forschung: Ansätze, Potentiale und Herausforderungen». In Forschungswerkstatt Medienpädagogik. Projekt - Theorie - Methode, herausgegeben von Thomas Knaus, 59-95. München: kopaed. https://doi.org/10.25656/01:17072.

Pietraß, Manuela. 2003. Bild und Wirklichkeit. Zur Unterscheidung von Realität und Fiktion bei der Medienrezeption. Opladen: Leske + Budrich.

Reichenbach, Roland, und Nicolaj van der Meulen. 2010. «Ästhetisches Urteil und Bildkompetenz. Einleitend zum Thementeil». Zeitschrift für Pädagogik 56 (6): 795-805. https://doi.org/10.25656/01:7306.

Reißmann, Wolfgang. 2015. Mediatisierung visuell. Kommunikationstheoretische Überlegungen und eine Studie zum Wandel privater Bildpraxis. Baden-Baden: Nomos. https://doi.org/10.5771/9783845265667.

Schlör, Katrin. 2017. «Subjektorientierte medienpädagogische Forschung mit Familien in belasteten Lebenslagen - Chancen und Herausforderungen visueller Forschungsmethoden». In Forschungswerkstatt Medienpädagogik. Projekt - Theorie - Methode, herausgegeben von Thomas Knaus, 287-315. München: kopaed. https://doi.org/10.25526/fw-mp.21.

Schulz-Zander, Renate. 2001. "Schulen ans Netz - aber wie? Die wirkungsvolle Einführung neuer Medien erfordert eine lernende Schule». Computer + Unterricht 41 (11), 6-9.

Shin, Wonsun, und Hyunjin Kang. 2016. «Adolescents' privacy concerns and information disclosure online: The role of parents and the Internet». Computers in Human Behavior 54:114-23. https://doi.org/10.1016/j.chb.2015.07.062.

Staksrud, Elisabeth. 2013. "Online grooming legislation: Knee-jerk regulation?». European Journal of Communication 28 (2): 152-67. https://doi. org/10.1177/0267323112471304.

Stubbe, Julian. 2016. «Material Practice as a Form of Critique». Interaction Design and Architecture(s) Journal - IxD\&A 30: 30-46. http://www.mifav.uniroma2.it/ inevent/events/idea2010/doc/30_2.pdf.

Stubbe, Julian. 2017. «Von digitaler zu soziodigitaler Souveränität». In Digitale Souveränität. Bürger | Unternehmen / Staat, herausgegeben von Volker Wittpahl, 43-59. Berlin, Heidelberg: Springer. https://doi.org/10.1007/978-3-66255796-9_1.

Thomas, Tanja, und Friedrich Krotz. 2008. «Medienkultur und soziales Handeln: Begriffsarbeiten zurTheorieentwicklung». In Medienkultur und soziales Handeln, herausgegeben von Tanja Thomas. 1. Aufl., 17-42. Medien - Kultur Kommunikation. Wiesbaden: VS Verlag für Sozialwissenschaften. https://doi. org/10.1007/978-3-531-90898-4_1. 
Tillmann, Angela. 2014. «Selfies. Selbst- und Körpererkundungen Jugendlicher in einer entgrenzten Gesellschaft». In Lieben, Liken, Spielen, herausgegeben von Jürgen Lauffer und Renate Röllecke, 42-51. München: kopaed.

Turkle, Sherry. 2011. Alone together: Why we expect more from technology and less from each other. New York: Basic Books. https://doi.org/10.1002/asi.22658.

Ulbing, Nora. 2020. "Das Phänomen Sexting - (k)ein Kinderspiel». Medien + Erziehung 64 (2): 60-66. https://www.merz-zeitschrift.de/alle-ausgaben/ details/2020-02-beruf-medienpaedagog-in/.

United Nations. 2021. General comment No. 25 on children's rights in relation to the digital environment. https://www.ohchr.org/EN/HRBodies/CRC/Pages/GCChildrensRightsRelationDigitalEnvironment.aspx.

United Nations Human Rights. 1989. "Convention on the Rights of the Child». https://www.ohchr.org/en/professionalinterest/pages/crc.aspx.

Vorderer, Peter, Dorothée Hefner, Leonard Reinecke, und Christoph Klimmt, Hrsg. 2018. Permanently Online, Permanently Connected: Living and Communicating in a POPC World. New York: Routledge. https://doi.org/10.4324/9781315276472.

Wagner, Ulrike, Niels Brüggen, und Christa Gebel. 2010. «Persönliche Informationen in aller Öffentlichkeit? Jugendliche und ihre Perspektive auf Datenschutz und Persönlichkeitsrechte in Sozialen Netzwerkdiensten». https://www.jff.de/ veroeffentlichungen/detail/persoenliche-informationen-in-aller-oeffentlichkeit-jugendliche-und-ihre-perspektive-auf-datenschutz/.

Wagner, Ulrike, Niels Brüggen, Peter Gerlicher, Mareike Schemmerling, und Christa Gebel. 2013. «Identitätsarbeit und sozialraumbezogenes Medienhandeln in Sozialen Netzwerkdiensten: Vierte Teilstudie der 5. Konvergenzstudie 〈Das Internet als Rezeptions- und Präsentationsplattform Jugendlicher〉》. Unveröffentlichtes Manuskript. https://www.jff.de/veroeffentlichungen/detail/identitaetsarbeit-und-sozialraumbezogenes-medienhandeln-in-sozialennetzwerkdiensteno/.

Wagner, Ulrike, und Susanne Eggert. 2013. «Das Medienhandeln von Heranwachsenden - Konstanten und Veränderungen: Materialen zum 14. Kinder- und Jugendbericht». https://www.dji.de/veroeffentlichungen/literatursuche/detailansicht/literatur/16304-das-medienhandeln-von-heranwachsenden-konstanten-und-veraenderungen.html.

Wagner, Ulrike, Christa Gebel, und Claudia Lampert, Hrsg. 2013. Zwischen Anspruch und Alltagsbewältigung: Medienerziehung in der Familie. Schriftenreihe Medienforschung der Landesanstalt für Medien Nordrhein-Westfalen 72. Berlin: Vistas. https://www.medienanstalt-nrw.de/zum-nachlesen/forschung/abgeschlossene-projekte/schriftenreihe-medienforschung/zwischen-anspruchund-alltagsbewaeltigung-medienerziehung-in-der-familie.html.

Wisniewski, Pamela. 2018. «The Privacy Paradox of Adolescent Online Safety: A Matter of Risk Prevention or Risk Resilience?». IEEE Secur. Privacy 16 (2): 8690. https://doi.org/10.1109/MSP.2018.1870874. 